Chinese Journal of Astronomy and Astrophysics manuscript no.

(ATEX: paper.tex; printed on February 2, 2008; 13:08)

\title{
Microquasars as high-energy gamma-ray sources
}

\author{
Josep M. Paredes * \\ Departament d'Astronomia i Meteorologia, Universitat de Barcelona, Av. Diagonal 647, \\ 08028 Barcelona, Spain \\ Received 2004 month day; accepted 2004 month day
}

\begin{abstract}
Galactic microquasars are certainly one of the most recent additions to the field of high energy astrophysics and have attracted increasing interest over the last decade. However, the high energy part of the spectrum of microquasars is the most poorly known, mainly due the lack of sensitive instrumentation in the past. Microquasars are now primary targets for all of the observatories working in the X-ray and gamma-ray domains. They also appear as the possible counterparts for some of the unidentified sources of high-energy gamma-rays detected by the experiment EGRET on board the satellite COMPTON-GRO. This paper provides a general review of the main observational results obtained up to now as well as a summary of the scenarios for production of high-energy $\gamma$-rays at the present moment.
\end{abstract}

Key words: X-rays: binaries - stars: individual: LS 5039, LS I +61 303 gamma-rays: observations

\section{INTRODUCTION}

The spectrum of microquasars extends from radio wavelengths to $\gamma$-rays (Mirabel \& Rodríguez 1999, Fender 2004). Therefore, a complete understanding of these objects necessarily requires a multi-wavelength approach using both ground and space-based telescopes and detectors. Such an effort is justified in view of the high interest raised by the quasar-microquasars connections. Microquasars provide an excellent laboratory for a suitable study of mass accretion and ejection phenomena in the strong gravitational field of a black hole or neutron star on human timescales. For instance, it has been possible to prove the direct connection between instabilities of the accretion disk and formation

$\star$ E-mail: jmparedes@ub.edu 
of jets in the microquasar and black hole candidate GRS $1915+105$ on timescales of minutes-hours (Fender et al.1997, Mirabel et al.1998). On the other hand, microquasars appear as a possible explanation for some of the unidentified sources of high-energy $\gamma$ rays detected by the experiment EGRET on board the satellite COMPTON-GRO. In particular, the microquasar LS 5039 is the best representative of the proposed connection between microquasars and unidentified EGRET sources (Paredes et al.2000), although it is not a unique case. LS I+61 303, whose microquasar nature was stablished when VLBI observations detected a relativistic radio jet (Massi et al. 2001), is also the possible counterpart of a high-energy $\gamma$-ray source (Gregory \& Taylor 1978, Kniffen et al. 1997). So, microquasars have turned out to be likely high-energy $\gamma$-ray emitting objects, being also interesting targets to be observed by the new generation of Cherenkov imaging telescopes at very high-energy $\gamma$-rays.

\section{THE MICROQUASAR POPULATION IN OUR GALAXY}

Microquasars, understood as X-ray Binaries with relativistic radio jets, represent a growing subset of the X-ray binary population in the Galaxy. The most recent catalogue of High Mass X-ray Binaries (HMXBs) contains 130 sources (Liu et al. 2000), while the catalogue of Low Mass X-ray Binaries (LMXBs) amounts to 150 objects (Liu et al. 2001). Considering both catalogues together, there are about 75 X-ray pulsars, which are not radio emitters, and a total of 43 radio emitting sources, some of which have been found to be microquasars (Ribó 2002, Ribó 2004). Recently, it has been estimated that the total number of X-ray Binaries in the Galaxy brighter than $2 \times 10^{34} \mathrm{erg} \mathrm{s}^{-1}$ is about 705, being distributed as $\sim 325$ LMXBs and $\sim 380$ HMXBs (Grimm et al. 2002). This suggests an upper limit on the population of microquasars in the Galaxy of about one hundred systems. A decade ago, one could count the known microquasars with the fingers of one hand. Now, the situation is much better but we do not have yet a microquasar population big enough from which statistically robust results can be derived.

At the time of writing, a total of 15 microquasar systems have been identified. All of them are listed in Table 1 The top part of the table is reserved for High Mass Xray Binaries, while the bottom part contains those of low mass. Within each group, the objects are sorted by right ascension and the following information is given: name and position; type of system; distance; orbital period; mass of the compact object; degree of activity (persistent/transient radio emission); apparent velocity of the ejecta; inclination and size of the jets; remarks.

There are some sources, e.g. XTE J1118+480, considered as microquasar candidates because their radio emission has not yet been resolved in relativistic jets, although their existence is inferred from a theoretical point of view. 
It is very likely that discoveries in the near future will increase substantially the galactic microquasar census and Table 1 will soon become obsolete. Similarly, nothing prevents us from thinking that microquasars in nearby galaxies could eventually be identified. However, such a detection will certainly be quite difficult due to the very large distances involved. Of course, such detection could become feasible provided that extreme relativistic beaming effects can occur (e.g. microblazars as possible ultraluminous X-ray sources).

\section{WHY SHOULD WE EXPECT MICROQUASARS TO BE $\gamma$-RAY EMITTERS?}

It is widely accepted that relativistic jets in AGNs are strong emitters of $\gamma$-rays with GeV energies (e.g. von Montigny et al.1995). Generally speaking, and allowing for their similarity (Mirabel \& Rodríguez 1999), one could also expect the jets in microquasars to be $\mathrm{GeV} \gamma$-ray emitters. In some cases, however, the sensitivity of the current $\gamma$-ray detectors may not be high enough to detect such emission. For instance, based on the physical parameters derived from observations of outbursts, the expected $\gamma$-ray flux of GRS 1915+105 up to very high-energy $\gamma$-rays has been estimated from inverse Compton scattering of the synchrotron photons (Atoyan \& Aharonian 1999). The resulting fluxes could have been hardly detected by EGRET, being also of transient nature, but they are within the sensitivity of the future AGILE and GLAST missions, about 10-100 times better than that of EGRET.

Several models have been developed to explore the high energy emission from the jets of microquasars. Two kinds of model can be found in the literature depending on whether hadronic or leptonic jet matter dominates the emission at such an energy range: the hadronic jet models (e.g. Romero et al. 2003), and the leptonic jet models. Among leptonic jet models, there are IC jet emission models that can produce X-rays and $\gamma$ rays, based in some cases on the synchrotron self-Compton (SSC) process (i.e. Band \& Grindlay 1986 Atoyan \& Aharonian 1999), and in other cases on external sources for the IC seed photons (EC) (i.e. Kaufman Bernadó et al.2002 Georganopoulos et al.2002). In addition, there are synchrotron jet emission models that can produce X-rays (i.e. Markoff et al. 2003). A general description of such models can be found in Romero (2004).

\section{HIGH ENERGY OBSERVATIONS}

In the next subsections we review and comment on the observational data of the microquasars listed in Table 1 at energies from soft to very high-energy $\gamma$-rays, and we quote them in Table 2 


\subsection{INTEGRAL, BATSE and COMPTEL sources}

Bird et al. (2004) have reported the first high-energy survey catalog obtained with the IBIS $\gamma$-ray imager on board INTEGRAL, covering the first year data. This initial survey has revealed the presence of $\sim 120$ sources detected with a good sensitivity in the energy range $20-100 \mathrm{keV}$. Among the detected sources we have inspected the microquasars listed in Table 1 In the second column of Table 2 we list their significance in the $30-50 \mathrm{keV}$ energy range and in the third column their flux (count/s) and error or upper limit in the energy range of $40-100 \mathrm{keV}$.

The Burst and Transient Source Experiment (BATSE), aboard the Compton Gamma Ray Observatory (CGRO), monitored the high energy sky using the Earth occultation technique (EOT). A compilation of BATSE EOT observations has been published recently (Harmon et al. 2004), with the flux data for the sample being presented in four energy bands. From this catalog we have selected also the data on microquasars. In the fourth column of Table the significance in the energy range 20-100 keV is listed, while in the fifth column we have listed their flux in the energy range $160-430 \mathrm{keV}$ in mCrab units. Cygnus X-1 and Cygnus X-3 have been studied extensively by BATSE.

The instrument COMPTEL, also aboard the CGRO, detected 32 steady sources and $31 \gamma$-ray bursters (Schönfelder et al. 2000). Among the continuum sources detected there are the microquasar Cygnus X-1 and other two sources, GRO J1823-12 and GRO J0241+6119, possibly associated with two other microquasars. See sixth column in Table 2

The standard interpretation of the emission in the low-energy $\gamma$-ray range is that disc blackbody photons are Comptonized by thermal/nonthermal electrons. There are state transitions (hard and low states) thought to be related to changes in the mass accretion rate. Nevertheless, it is still unclear whether this is what really happens. Alternatively, some groups have suggested that this emission could come from the jet, basing this idea on recent observational and theoretical results (see, i.e., Fender et al. 2003, Markoff et al. 2003, Georganopoulos et al. 2002).

\subsection{EGRET sources}

\subsubsection{LS 5039 / 3EG J1824-1514}

The discovery of the microquasar LS 5039, and its possible association with a highenergy $\gamma$-ray source $(E>100 \mathrm{MeV})$, provides observational evidence that microquasars could also be sources of high-energy $\gamma$-rays (Paredes et al. 2000). It is important to point out that this is the first time that an association between a microquasar and a high-energy $\gamma$-ray source has been reported. This finding opens up the possibility that other unidentified EGRET sources could also be microquasars. LS 5039 is the only X-ray 


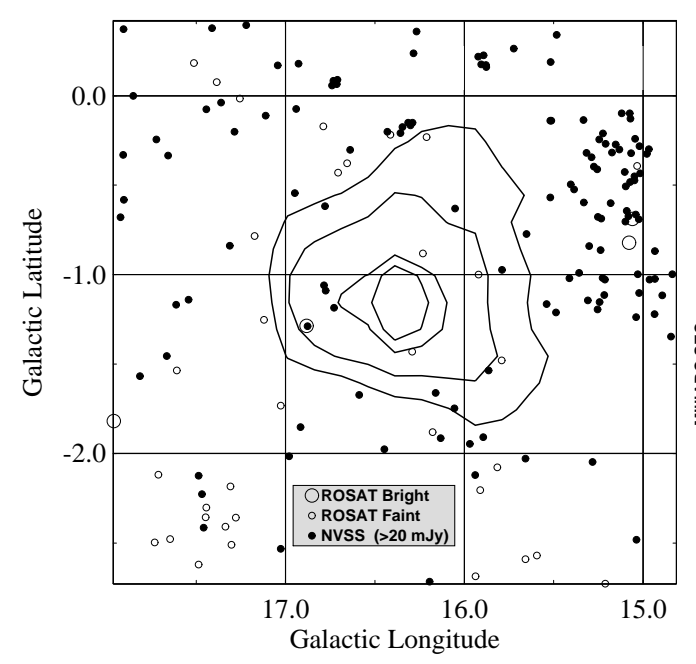

Fig. 1 Location map of the EGRET source 3EG J1824-1514. The contours represent $50 \%, 68 \%, 95 \%$ and $99 \%$ statistical probability that a $\gamma$-ray source lies within the given contour. The only X-ray and radio source is LS 5039 (filled circle inside an open big circle at $\mathrm{l}=16.66^{\circ}$ and $\mathrm{b}=-1.29^{\circ}$ ) (Ribó 2002).

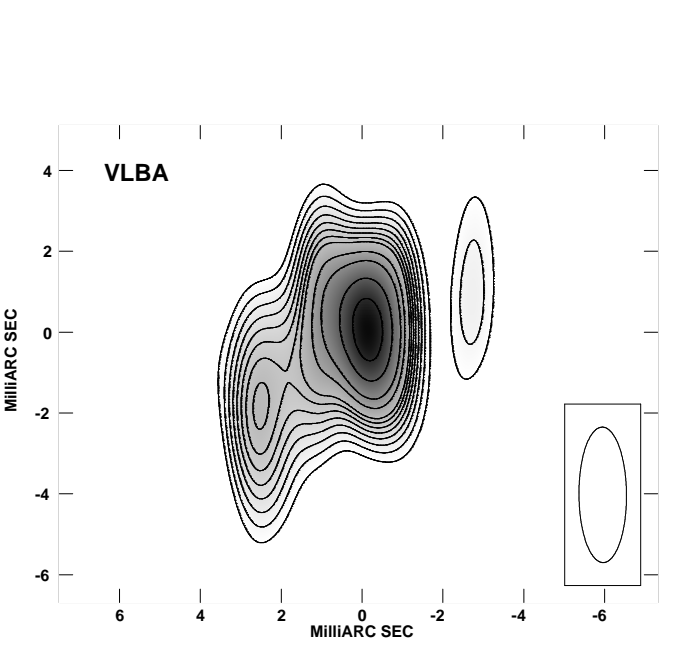

Fig. 2 Relativistic radio jets of LS 5039, observed with the VLBA, which reveal its microquasar nature (Paredes et al. 2000).

source from the bright ROSAT catalog whose position is consistent with the high energy $\gamma$-ray source 3EG J1824-1514. LS 5039 is also the only object simultaneously detected

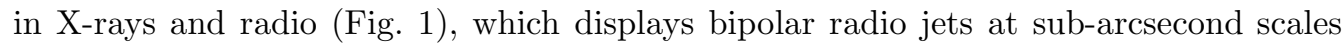
(Fig. 2). New observations conducted with the EVN and MERLIN confirm the presence of an asymmetric two-sided jet reaching up to $\sim 1000 \mathrm{AU}$ on the longest jet arm (Fig. 3) (Paredes et al. 2002, Ribó 2002).

Recently, Collmar (2003) has reported the detection of an unidentified $\gamma$-ray source, GRO J1823-12, at galactic coordinates $\left(l=17.5^{\circ}, \mathrm{b}=-0.5^{\circ}\right)$ by the COMPTEL experi-

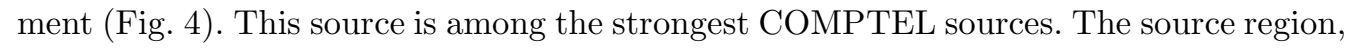
detected at high significance level, contains several possible counterparts, being LS 5039 one of them. It is also worth noting that BATSE has detected this source at soft $\gamma$-rays (see Table 2). Taking into account these observational evidences, from radio to highenergy $\gamma$-rays, LS 5039 appears to be a very likely counterpart of the EGRET source 3EG J1824-1514.

Regarding the theoretical point of view, the $\gamma$-ray emission from 3EG J1824-1514, with a luminosity of $\mathrm{L}_{\gamma}(>100 \mathrm{MeV}) \sim 10^{35} \mathrm{erg} \mathrm{s}^{-1}$, is likely to be originated from inverse Compton effect of the ultraviolet photons from a hot companion star scattered by the same relativistic electrons responsible for the radio emission (Paredes et al. 2000, Paredes 

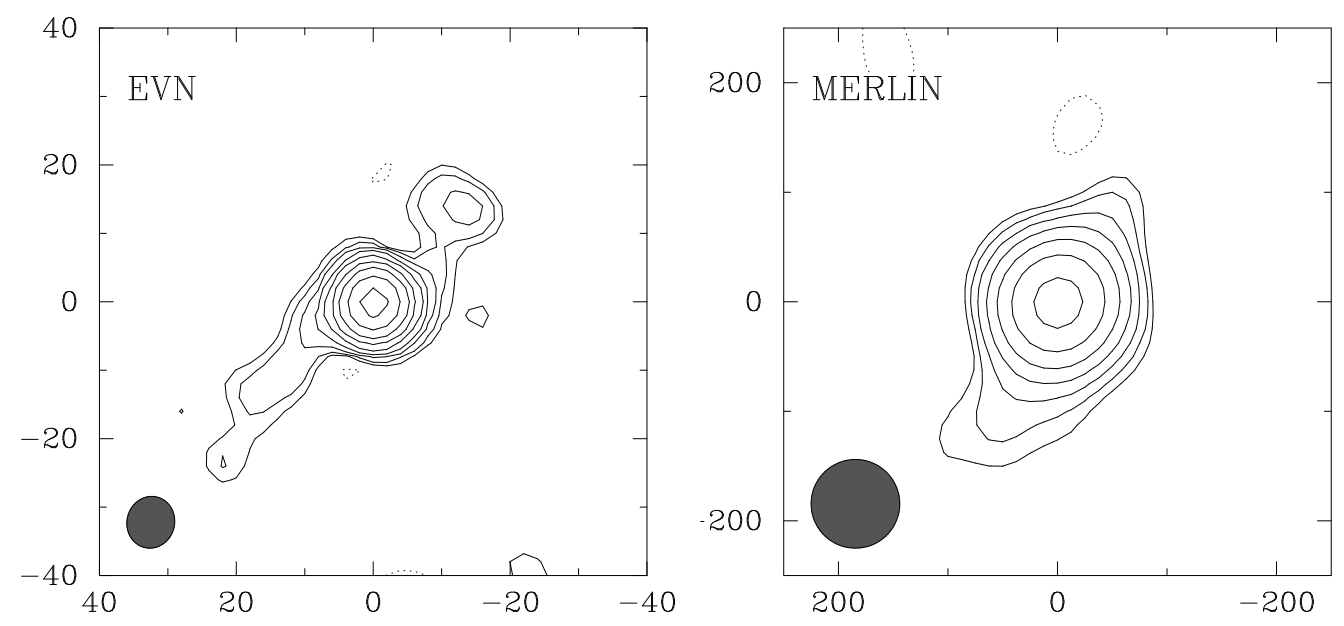

Fig. 3 Self-calibrated images of LS 5039 at 5 GHz obtained on March 12000 with the EVN (left) and MERLIN (right). Axis units are in milliarc-seconds (Paredes et al. 2002).

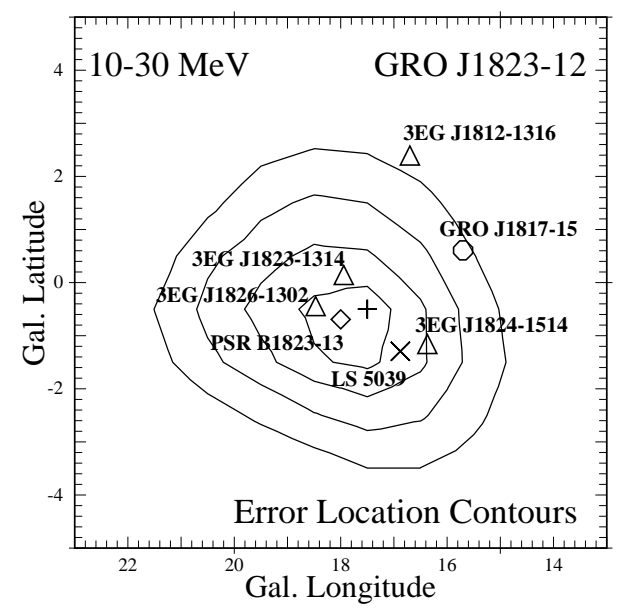

Fig. 4 Error location contours for the COMPTEL unidentified source GRO J1823-12. The most likely source position (+) and possible counterparts are overlaid (Collmar 2003).

et al. 2002). The energy shift in this process is given by $E_{\gamma} \sim \gamma_{\mathrm{e}}^{2} E_{\mathrm{ph}}$, where the energies of the $\gamma$-ray and the stellar photons are related by the Lorentz factor of the electrons squared. For an O6.5 star in the main sequence, such as the component of LS 5039, most of its luminosity is radiated by photons with $E_{\mathrm{ph}} \sim 10 \mathrm{eV}$. To scatter them into $\gamma$-ray photons with $E_{\gamma} \sim 100 \mathrm{MeV}$, electrons with a Lorentz factor of $\gamma_{\mathrm{e}} \sim 10^{4}$, or equivalently with energy $\sim 10^{-2} \mathrm{erg}$, are required. A detailed numerical model to explain the $\gamma$-ray emission of LS 5039 has been proposed recently (Bosch-Ramon \& Paredes 2004a). 


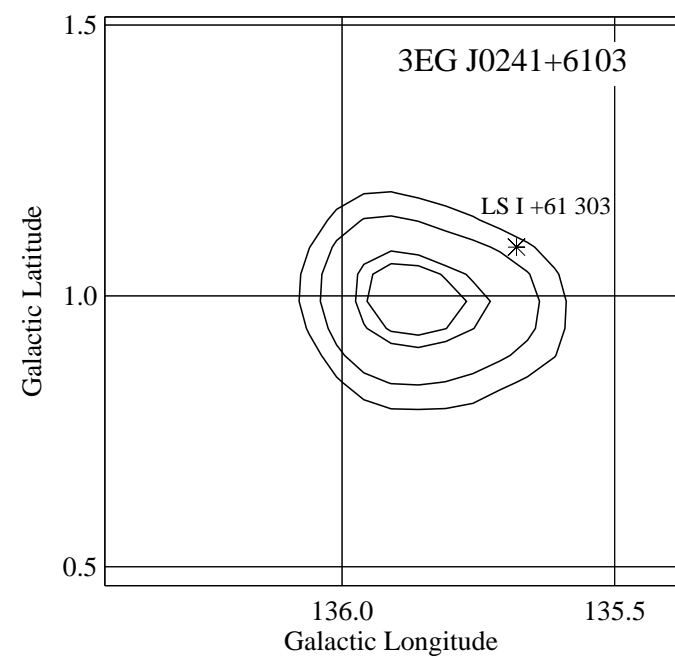

Fig. 5 Location map for 3EG J0241+6103 in the 3rd EGRET catalog (adapted from Hartman et al. 1999).

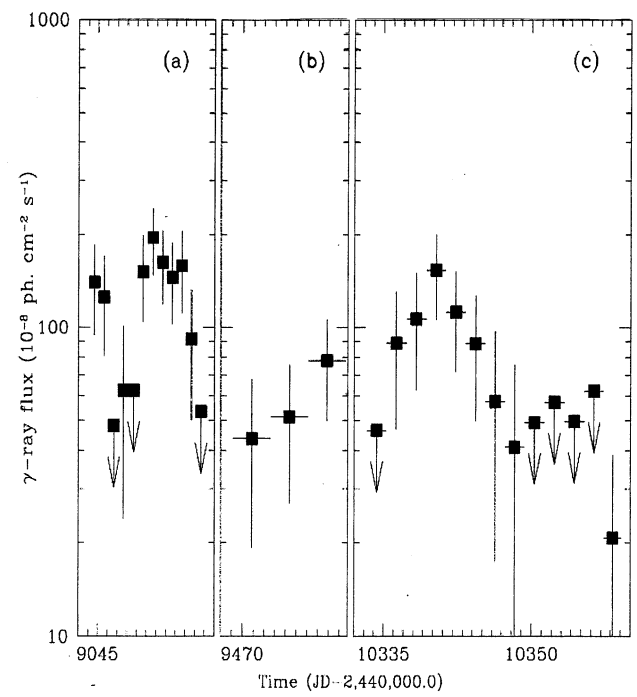

Fig. 6 EGRET light curve of 2CG 135+01 / 3EG J0241+6103 for the best near-axis observations (Tavani et al. 1998).

\subsection{2 $L S I+61303 / 3 E G J 0241+6103$}

The well known High Mass X-ray binary LS I +61 303 has been classified as a new microquasar after the discovery of relativistic jets (Massi et al. 2001, Massi et al. 2004). This object has also been proposed to be associated with the $\gamma$-ray source 2CG $135+01$ (=3EG J0241+6103) (Gregory \& Taylor 1978, Kniffen et al.1997). Although the broadband $1 \mathrm{keV}-100 \mathrm{MeV}$ spectrum of LS I +61 303 remains uncertain, because OSSE and COMPTEL observations were likely dominated by the quasar QSO 0241+622 emission, the EGRET angular resolution is high enough to exclude this quasar as the source of the $\gamma$-ray emission (Harrison et al. 2000). BATSE marginally detected the source, being the quasar also excluded as the origin of this emission (see Table 2). We show the location map of 3EG J0241+6103 in Fig. (Hartman et al. 1999).

A timing analysis carried out recently by Massi (2004) of pointed EGRET observations (Tavani et al. 1998) (Fig. 6) suggests a period of $27.4 \pm 7.2$ days, in agreement with the orbital period of this binary system, of 26.496 days. This result, if confirmed, would clearly support the association of LS I +61 303 with 3EG J0241+6103.

This microquasar also seems to be a fast precessing system. MERLIN images obtained in two consecutive days (Fig. (7) show a change in the direction of the jets of about $50^{\circ}$ that has been interpreted as a fast precession of the system (Massi et al. 2004). If this is confirmed, it could solve the puzzling VLBI structures observed so far, as well as the short term variability of the associated $\gamma$-ray source 3EG J0241+6103 (e.g. Wallace et al. [2000). 

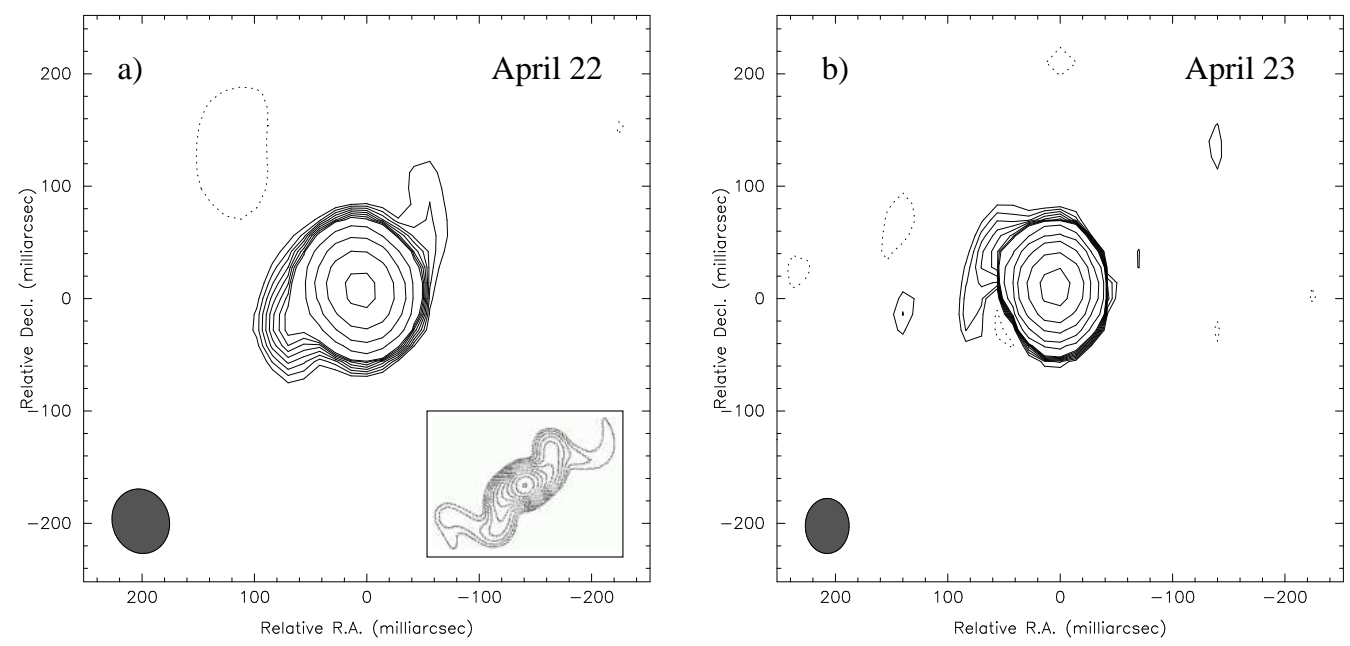

Fig. 7 MERLIN self-calibrated images of LS I +61303 at $5 \mathrm{GHz}$ obtained on April 22 (left) and April 23 (right). The S-shaped morphology recalls the precessing jet of SS 433, whose simulated radio emission is given in the small box (Massi et al. 2004).

Up to now, the only existing radial velocity curve of LS I +61 303 was that obtained by Hutchings and Crampton (1981). Recently, after a spectroscopic campaign, an improved estimation of the orbital parameters has been obtained (Casares et al. 2004). Here, we will just mention the new high eccentricity $(\mathrm{e}=0.72 \pm 0.15)$ and the periastron orbital phase at $\sim 0.2$. These values are a key information for any interpretation of the data obtained at any wavelength.

Hall et al. (2003) gave upper limits for the emission associated to LS I +61 303 / 3EG J0241+6103 at very high-energy $\gamma$-rays from observations performed by the Cherenkov telescope Whipple (Fig [ 8 ). Several models have been proposed to explore the high energy emission of this source (e.g. Taylor et al. 1996. Punsly 1999. Harrison et al. 2000, Leahy 2004). The most recent model has been presented by Bosch-Ramon $\&$ Paredes (2004b), who explore with a detailed numerical model if this system can produce the emission and to present the variability detected by EGRET ( $>100 \mathrm{MeV})$. We reproduce in Fig. 9 the computed spectral photon distribution, which is able to fit the data.

\subsubsection{Other EGRET/microquasar candidates}

After a multiwavelength study of the X-ray source AX J1639.0-4642, Combi et al. (2004) have proposed that this source is a dust-enshrouded microquasar candidate. This source is within the $95 \%$ location contours of 3EG J1639-4702 and has been proposed by the same authors to be the counterpart of the EGRET source. If confirmed, this would be 


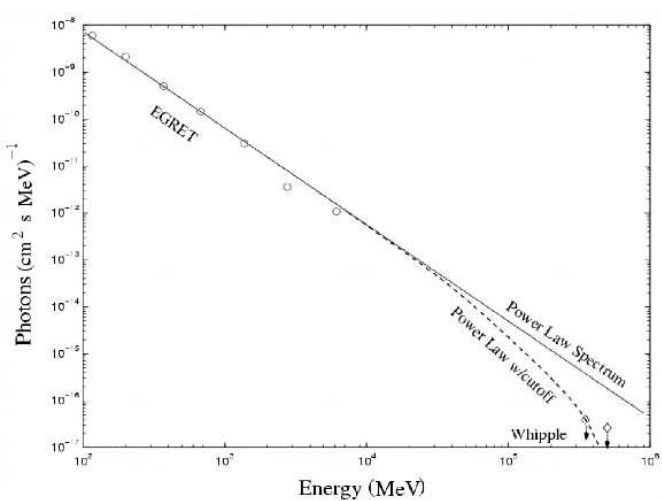

Fig. 8 EGRET data points of 3EG J0241+6103 (circles) and the flux upper limits (diamonds) obtained by Whipple (Hall et al. 2003).

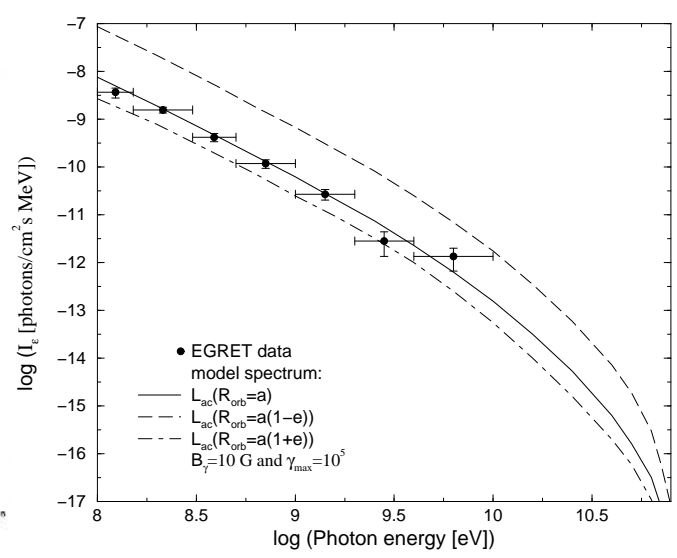

Fig. 9 Computed spectral photon distribution of LS I +61 303 above 100 $\mathrm{MeV}$ plotted with the EGRET data points of 3EG J0241+6103 (Bosch-Ramon \& Paredes 2004b).

an important result, although by now there are other possibilities such as the presence of the pulsar PSR J1637-4642 within the 95\% confidence contours (Torres et al. 2001).

\subsubsection{EGRET candidate microquasars as runaway objects}

A hot recent topic in the microquasar field is the measurement of high spatial velocities for some of these objects (the so-called runaway microquasars). Regarding the two microquasars that are EGRET source candidates, interesting kinematic properties have been observed. LS 5039, with a systemic velocity of $150 \mathrm{~km} \mathrm{~s}^{-1}$, is escaping from its local environment with a very high velocity component perpendicular to the Galactic Plane (Ribó et al. 2002). Such behaviour may be the result of the supernova explosion which created the compact object in this binary system. According to the computed trajectory and the possible lifetime of the donor, LS 5039 could reach a galactic latitude of $-12^{\circ}$ and still behave as a microquasar. Taking into account the possible association of this microquasar with the high-energy $\gamma$-ray source 3EG J1824-1514, one could expect to detect gamma-ray microquasars up to \pm 10 degrees of galactic latitudee. Also LS I +61 303 is running away from its birth place with a linear momentum of $430 \pm 140 M_{\odot} \mathrm{km} \mathrm{s}^{-1}$, which is comparable to the linear momentum found in solitary runaway neutron stars (Mirabel et al. 2004). Other microquasars such as Scorpius X-1 and GRO J1655-40, or the microquasar candidate XTE J1118+480 (Mirabel et al.2001), display also significant space velocities, although so far they are not high-energy $\gamma$-ray source candidates. In general, as suggested by Romero et al. (2004), some microquasars with high spatial velocity 
could be related to the faint, soft and variable unidentified EGRET sources above and below the Galactic Plane.

\subsection{VHE $\gamma$-ray sources}

The very high energy sky map contains a reduced number of sources. The amount of confirmed and probable catalogued sources is presently fourteen (6 AGN, 3 pulsar wind nebulae, 3 supernova remnants, 1 starburst galaxy, and 1 unknown) (Ong 2003). Some microquasars have been observed in the energy range of $\mathrm{TeV} \gamma$-rays with the imaging atmospheric Cherenkov telescopes, but none of them has been detected with high confidence up to now. Historically, Cygnus X-3 was widely observed with the first generation of $\mathrm{TeV}$ instruments. Some groups claimed that they had detected Cygnus X-3 (Chadwick et al. 1985) whereas other groups failed to detect it (O'Flaherty et al. 1992). As the claimed detections have not been confirmed, and the instrumentation at this epoch was limited, these results have not been considered as definite positive detections by the community. The HEGRA experiment detected a flux of the order of 0.25 Crab from GRS 1915+105 during the period May-July 1996 when the source was in an active state (Aharonian \& Heinzelmann 1997). This source has also been observed with Whipple, obtaining a 3.1 $\sigma$ significance (Rovero et al.2002). More recently, an upper-limit of $0.35 \mathrm{Crab}$ above $400 \mathrm{GeV}$ has been quoted for GRS 1915+105 (Horan \& Weekes 2003). LS I +61 303 was observed too, and not detected in the TeV energy range (see Section 4.2.2).

\section{SUMMARY}

Microquasars are among the most interesting sources in the Galaxy from the viewpoint of high-energy astrophysics. Models predict that radio jets could be natural sites for the production of high-energy photons via both Compton scattering and maybe direct synchrotron emission (although hadronic emission is not discarded either). Although most microquasars have been detected as soft $\gamma$-ray sources, up to now only a few of them seem to be high-energy $\gamma$-ray emitters. Future missions such like AGILE and GLAST will confirm or reject the proposed association between some microquasars and EGRET sources. Also, observations of microquasars with the new generation of Cherenkov telescopes (MAGIC, H.E.S.S.) will bring more constraints to the physics of these systems.

Acknowledgements I am grateful to Marc Ribó, Valentí Bosch-Ramon and Josep Martí for their useful comments and suggestions while writing this paper. I also acknowledge partial support by DGI of the Ministerio de Ciencia y Tecnología (Spain) under grant AYA2001-3092, as well as partial support by the European Regional Development Fund (ERDF/FEDER). 


\section{References}

Aharonian, F.A., Heinzelmann, G., 1997, Proc. 15th European Cosmic Ray Symposium 1996 astro-ph/9702059

Atoyan, A.M., Aharonian, F.A., 1999, MNRAS, 302, 253

Band, D. L. \& Grindlay, J. E. 1986, ApJ, 311, 595

Bird, A.J., Barlow, E.J., Bassani, L., et al., 2004, ApJ, 607, L33

Bosch-Ramon, V., Paredes, J.M., 2004a, A\&A, 417, 1075

Bosch-Ramon, V., Paredes, J.M., 2004b, A\&A, in press, astro-ph/0407016

Casares, J., Ribas, I., Paredes, J., Martí, J., 2004, MNRAS, submitted

Chadwick, P.M., Dipper, N.A., Dowthwaite, J.C., et al., 1985, Nature, 318, 642

Collmar, W., 2003, Proc. 4th Agile Science Workshop, Frascati (Rome) on 11-13 June 2003

Combi, J.A., Ribó, M., Mirabel, I.F., Sugizaki, M., 2004, A\&A, in press, astro-ph/0401643

Fender, R. P. 2004, in 'Compact Stellar X-Ray Sources', eds. W. H. G. Lewin and M. van der Klis, Cambridge University Press, in press astro-ph/0303339

Fender, R. P., Pooley, G. G., Brocksopp, C., \& Newell, S. J. 1997, MNRAS, 290, L65

Fender, R. P., Gallo, E., \& Jonker, P. G. 2003, MNRAS, 343, L99

Georganopoulos, M., Aharonian, F. A., \& Kirk, J. G. 2002, A\&A, 388, L25

Gregory, P. C. \& Taylor, A. R. 1978, Nature, 272, 704

Grimm, H. J., Gilfanov, M., Sunyaev, R., 2002, A\&A, 391, 923

Hall, T.A., Bond, I.H., Bradbury, S.M., et al., 2003, ApJ, 583, 853

Harmon, B.A., Wilson, C.A., Fishman, G.J., et al., 2004, ApJS, in press astro-ph/0404453

Harrison, F.A., Ray, P.S., Leahy, D.A., et al., 2000, ApJ, 528454

Hartman, R.C., Bertsch, D.L., Bloom, S.D., et al., 1999, ApJS, 12379

Horan, D., Weekes, T., 2003, private communication

Hutchings, J.B., Crampton, D., 1981, PASP, 93, 486

Kaufman Bernadó, M. M., Romero, G. E., \& Mirabel, I. F. 2002, A\&A, 385, L10-L13

Kniffen, D.A., Alberts, W.C.K., Bertsch, D.L., et al., 1997, ApJ, 486, 126

Leahy, D.A., 2004, A\&A, 413, 1019

Liu, Q.Z., van Paradijs, J., van den Heuvel, E.P.J. 2000, A\&AS, 147, 25

Liu, Q.Z., van Paradijs, J., van den Heuvel, E.P.J. 2001, A\&A, 368, 1021

Markoff, S., Nowak, M., Corbel, S., Fender, R., \& Falcke, H. 2003, A\&A, 397, 645

Massi, M., Ribó, M., Paredes, J.M., Peracaula, M., Estalella, R., 2001, A\&A, 376, 217

Massi, M., Ribó, M., Paredes, J.M., Garrington, S.T., Peracaula, M., Martí, J., 2004, A\&A, 414, L1

Massi, M., 2004, A\&A, 422, 267

Mirabel, I. F., Dhawan, V., Chaty, S., et al. 1998, A\&A, 330, L9

Mirabel, I.F., Rodríguez, L.F., 1999 ARA\&A, 37, 409

Mirabel, I.F., Dhawan, V., Mignani, R.P., 2001, Nature, 413, 139

Mirabel, I.F., Rodrigues, I., Liu, Q.Z., 2004, A\&A, 422, L29

O'Flaherty, K.S., Cawley, M.F., Fegan, D.J., et al., 1992, ApJ, 396, 674

Ong, R. E. 2003, in proceedings of 'The Universe Viewed in Gamma-Rays', ed. R. Enomoto, Universal Academy Press Inc., Tokyo, in press, astro-ph/0304336

Paredes, J.M., Martí, J., Ribó, M., \& Massi, M., 2000, Science, 288, 2340

Paredes, J.M., Ribó, M., Ros, E., Martí, J., Massi, M., 2002, A\&A, 393, L99

Punsly, B., 1999, ApJ, 519, 336

Ribó, M., 2002, PhD Thesis, Universitat de Barcelona

Ribó, M., Paredes, J. M., \& Romero, G. E., et al. 2002, A\&A, 384, 954

Ribó, M. 2004, in ASP Conference Series: "Future Directions in High Resolution Astronomy: A Celebration of the 10th Anniversary of the VLBA", J. D. Romney \& M. J. Reid (eds.), astro-ph/0402134

Romero, G.E. et al., 2003, A\&A, 410, L1 
Romero, G. E., Grenier, I. A., Kaufman Bernadó, M., Mirabel, I. F., \& Torres, D. F. 2004, in proceedings of the 'Fifth INTEGRAL workshop The Integral Universe', ESA SP, in press, astro-ph/0402285

Romero G.E., 2004, ChJAA, in preparation

Rovero, A.C., Fegan, S., Weekes, T.C., 2002, BAAA, 45, 66

Schönfelder, V., Bennett, K., Blom, J.J., et al., 2000, A\&AS, 143, 145

Tavani et al., 1998, ApJ, 497, L89

Taylor, A.R., Young, G., Peracaula, M., et al., 1996, A\&A, 305, 817

Torres, D.F., Butt, Y.M., Camilo, F., 2001, ApJ, 560, L155

von Montigny, C., Bertsch, D.L., Chiang, J., et al., 1995, ApJ, 440, 525

Wallace, P. M., Griffis, N. J., Bertsch, D. L., et al. 2000, ApJ, 540, 184 
Table 1 Microquasars in our Galaxy

\begin{tabular}{|c|c|c|c|c|c|c|c|c|c|c|}
\hline Name & $\begin{array}{l}\text { Position } \\
\text { (J2000.0) }\end{array}$ & $\begin{array}{l}\text { System } \\
\text { type }\end{array}$ & $\begin{array}{c}D \\
(\mathrm{kpc})\end{array}$ & $\begin{array}{l}P_{\text {orb }} \\
\text { (d) }\end{array}$ & $\begin{array}{c}M_{\text {compact }} \\
\left(M_{\odot}\right)\end{array}$ & $\begin{array}{l}\text { Activity } \\
\text { radio }^{(b)}\end{array}$ & $\beta_{\text {apar }}$ & $\theta^{(\mathrm{c})}$ & $\begin{array}{c}\text { Jet size } \\
(\mathrm{AU})\end{array}$ & $\operatorname{Remarks}^{(\mathrm{d})}$ \\
\hline \multicolumn{11}{|c|}{ High Mass X-ray Binaries (HMXB) } \\
\hline \multirow[t]{2}{*}{ LS I +61 303} & $02^{\mathrm{h}} 40^{\mathrm{m}} 31^{\mathrm{s}} \cdot 66$ & B0V & 2.0 & 26.5 & - & $\mathrm{p}$ & $\geq 0.4$ & - & $10-700$ & Prec? \\
\hline & $+61^{\circ} 13^{\prime} 45^{\prime \prime} 6$ & $+\mathrm{NS} ?$ & & & & & & & & \\
\hline \multirow[t]{2}{*}{ V4641 Sgr } & $18^{\mathrm{h}} 19^{\mathrm{m}} 21^{\mathrm{s}} \cdot 48$ & B9III & $\sim 10$ & 2.8 & 9.6 & $\mathrm{t}$ & $\geq 9.5$ & - & - & \\
\hline & $-25^{\circ} 25^{\prime} 36^{\prime \prime} 0$ & $+\mathrm{BH}$ & & & & & & & & \\
\hline \multirow[t]{2}{*}{ LS 5039} & $18^{\mathrm{h}} 26^{\mathrm{m}} 15^{\mathrm{s}} .05$ & $\mathrm{O} 6.5 \mathrm{~V}((\mathrm{f}))$ & 2.9 & 4.4 & $1-3$ & $\mathrm{p}$ & $\geq 0.15$ & $<81^{\circ}$ & $10-1000$ & Prec? \\
\hline & $-14^{\circ} 50^{\prime} 54^{\prime \prime} 24$ & $+\mathrm{NS} ?$ & & & & & & & & \\
\hline \multirow[t]{2}{*}{ SS 433} & $19^{\mathrm{h}} 11^{\mathrm{m}} 49.6$ & evolved A? & 4.8 & 13.1 & $11 \pm 5 ?$ & $\mathrm{p}$ & 0.26 & $79^{\circ}$ & $\sim 10^{4}-10^{6}$ & Prec \\
\hline & $+04^{\circ} 58^{\prime} 58^{\prime \prime}$ & $+\mathrm{BH} ?$ & & & & & & & & XRJ \\
\hline \multirow[t]{2}{*}{ Cygnus X-1 } & $19^{\mathrm{h}} 58^{\mathrm{m}} 21^{\mathrm{s}} \cdot 68$ & O9.7Iab & 2.5 & 5.6 & 10.1 & $\mathrm{p}$ & - & $40^{\circ}$ & $\sim 40$ & \\
\hline & $+35^{\circ} 12^{\prime} 05^{\prime \prime}{ }^{\prime} 8$ & $+\mathrm{BH}$ & & & & & & & & \\
\hline \multirow[t]{2}{*}{ Cygnus X-3 } & $20^{\mathrm{h}} 32^{\mathrm{m}} 25^{\mathrm{s}} \cdot 78$ & WNe & 9 & 0.2 & - & $\mathrm{p}$ & 0.69 & $73^{\circ}$ & $\sim 10^{4}$ & \\
\hline & $+40^{\circ} 57^{\prime} 28^{\prime \prime} 0$ & $+\mathrm{BH} ?$ & & & & & & & & \\
\hline
\end{tabular}

Low Mass X-ray Binaries (LMXB)

\begin{tabular}{|c|c|c|c|c|c|c|c|c|c|c|}
\hline \multirow[t]{2}{*}{ Circinus X-1 } & $15^{\mathrm{h}} 20^{\mathrm{m}} 40^{\mathrm{s}} \cdot 9$ & Subgiant & 5.5 & 16.6 & - & $\mathrm{t}$ & $>15$ & $<6^{\circ}$ & $>10^{4}$ & \\
\hline & $-57^{\circ} 10^{\prime} 01^{\prime \prime}$ & $+\mathrm{NS}$ & & & & & & & & \\
\hline \multirow[t]{2}{*}{ XTE J1550-564 } & $415^{\mathrm{h}} 50^{\mathrm{m}} 58^{\mathrm{s}} .70$ & G8-K5V & 5.3 & 1.5 & 9.4 & $\mathrm{t}$ & $>2$ & - & $\sim 10^{3}$ & XRJ \\
\hline & $-56^{\circ} 28^{\prime} 35^{\prime \prime} 2$ & $+\mathrm{BH}$ & & & & & & & & \\
\hline \multirow[t]{2}{*}{ Scorpius X-1 } & $16^{\mathrm{h}} 19^{\mathrm{m}} 55^{\mathrm{s}} 1$ & Subgiant & 2.8 & 0.8 & 1.4 & $\mathrm{p}$ & 0.68 & $44^{\circ}$ & $\sim 40$ & \\
\hline & $-15^{\circ} 38^{\prime} 25^{\prime \prime}$ & $+\mathrm{NS}$ & & & & & & & & \\
\hline \multirow[t]{2}{*}{ GRO J1655-40 } & $16^{\mathrm{h}} 54^{\mathrm{m}} 00^{\mathrm{s}} \cdot 25$ & F5IV & 3.2 & 2.6 & 7.02 & $\mathrm{t}$ & 1.1 & $72^{\circ}-85^{\circ}$ & 8000 & Prec? \\
\hline & $-39^{\circ} 50^{\prime} 45^{\prime \prime} 0$ & $+\mathrm{BH}$ & & & & & & & & \\
\hline \multirow[t]{2}{*}{ GX 339-4 } & $17^{\mathrm{h}} 02^{\mathrm{m}} 49^{\mathrm{s}} .5$ & - & $>6$ & 1.76 & $5.8 \pm 0.5$ & $\mathrm{t}$ & - & - & $<4000$ & \\
\hline & $-48^{\circ} 47^{\prime} 23^{\prime \prime}$ & $+\mathrm{BH}$ & & & & & & & & \\
\hline \multirow[t]{2}{*}{ 1E $1740.7-2942$} & $17^{\mathrm{h}} 43^{\mathrm{m}} 54^{\mathrm{s}} .83$ & - & $8.5 ?$ & $12.5 ?$ & - & $\mathrm{p}$ & - & - & $\sim 10^{6}$ & \\
\hline & $-29^{\circ} 44^{\prime} 42^{\prime \prime} 60$ & $+\mathrm{BH} ?$ & & & & & & & & \\
\hline \multirow[t]{2}{*}{ XTE J1748-288 } & $17^{\mathrm{h}} 48^{\mathrm{m}} 05^{\mathrm{s}} 06$ & - & $\geq 8$ & $?$ & $>4.5 ?$ & $\mathrm{t}$ & 1.3 & - & $>10^{4}$ & \\
\hline & $-28^{\circ} 28^{\prime} 25^{\prime \prime} 8$ & $+\mathrm{BH} ?$ & & & & & & & & \\
\hline \multirow[t]{2}{*}{ GRS $1758-258$} & $18^{\mathrm{h}} 01^{\mathrm{m}} 12^{\mathrm{s}} \cdot 40$ & - & $8.5 ?$ & $18.5 ?$ & - & $\mathrm{p}$ & - & - & $\sim 10^{6}$ & \\
\hline & $-25^{\circ} 44^{\prime} 36^{\prime \prime} 1$ & $+\mathrm{BH} ?$ & & & & & & & & \\
\hline \multirow[t]{2}{*}{ GRS $1915+105$} & $19^{\mathrm{h}} 15^{\mathrm{m}} 11^{\mathrm{s}} .55$ & $\mathrm{~K}-\mathrm{M}$ III & 12.5 & 33.5 & $14 \pm 4$ & $\mathrm{t}$ & $1.2-1$. & $766^{\circ}-70^{\circ}$ & $\sim 10-10^{4}$ & Prec? \\
\hline & $+10^{\circ} 56^{\prime} 44^{\prime \prime} 7$ & $+\mathrm{BH}$ & & & & & & & & \\
\hline
\end{tabular}

Notes: ${ }^{\text {(a) }}$ NS: neutron star; BH: black hole. ${ }^{(b)}$ p: persistent; t:transient. ${ }^{\text {(c) }}$ jet inclination. ${ }^{(d)}$

Prec: precession; XRJ: X-ray jet. 
Table 2 High energy emission from microquasars

\begin{tabular}{|c|c|c|c|c|c|}
\hline \multirow[t]{2}{*}{ Name } & INTEGRAL $^{(\mathrm{a})}$ & $\operatorname{BATSE}^{(\mathrm{b})}$ & COMPTEL $^{(\mathrm{c})}$ & EGRET $^{(\mathrm{d})}$ & Others $^{(\mathrm{e})}$ \\
\hline & $\begin{array}{l}30-50 \mathrm{keV} \quad 40-100 \mathrm{keV} \\
\text { (significance) }(\text { count } / \mathrm{s} \text { ) }\end{array}$ & $\begin{array}{l}20-100 \mathrm{keV} 160-430 \mathrm{keV} \\
\text { (significance) } \quad \text { (mCrab) }\end{array}$ & $\begin{array}{c}1-30 \mathrm{MeV} \\
(\mathrm{GRO})\end{array}$ & $\begin{array}{c}>100 \mathrm{MeV} \\
(3 \mathrm{EG})\end{array}$ & \\
\hline
\end{tabular}

High Mass X-ray Binaries (HMXB)

\begin{tabular}{lcccccc}
\hline LS I +61 303 & - & - & 5.2 & $5.1 \pm 2.1$ & J0241+6119? J0241+6103? \\
V4641 Sgr & - & - & - & - & - & - \\
LS 5039 & - & - & 10.7 & $3.7 \pm 1.8$ & J1823-12? & J1824-1514? \\
SS 433 & 13.5 & $<1.02$ & 21.7 & $0.0 \pm 2.8$ & - & - \\
Cygnus X-1 & 676.6 & $66.4 \pm 0.1$ & 1186.8 & $924.5 \pm 2.5$ & yes & - \\
Cygnus X-3 & 122.7 & $5.7 \pm 0.1$ & 197.8 & $15.5 \pm 2.1$ & - & S \\
\hline
\end{tabular}

Low Mass X-ray Binaries (LMXB)

\begin{tabular}{|c|c|c|c|c|c|c|c|}
\hline Circinus X-1 & - & - & 3.8 & $0.3 \pm 2.6$ & - & - & \\
\hline XTE J1550-564 & 8.6 & $0.6 \pm 0.07$ & 17.1 & $-2.3 \pm 2.5$ & - & - & \\
\hline Scorpius X-1 & 111.6 & $2.3 \pm 0.1$ & 460.6 & $9.9 \pm 2.2$ & - & - & \\
\hline GRO J1655-40 & - & - & 40.6 & $23.4 \pm 3.9$ & - & - & $\mathrm{O}$ \\
\hline GX 339-4 & 21.9 & $0.55 \pm 0.03$ & 89.0 & $580 \pm 3.5$ & - & - & $\mathrm{S}$ \\
\hline 1E $1740.7-2942$ & 147.3 & $4.32 \pm 0.03$ & 92.4 & $61.2 \pm 3.7$ & - & - & $\mathrm{S}$ \\
\hline XTE J1748-288 & - & - & -12.4 & - & - & - & $\mathrm{S}$ \\
\hline GRS 1758-258 & 135.9 & $3.92 \pm 0.03$ & 74.3 & $38.0 \pm 3.0$ & - & - & $\mathrm{S}$ \\
\hline GRS $1915+105$ & 144.9 & $8.63 \pm 0.13$ & 208.8 & $33.5 \pm 2.7$ & - & - & $\mathrm{S}, \mathrm{T} ?$ \\
\hline
\end{tabular}

Notes: (a) The first IBIS/ISGRI soft gamma-ray galactic plane survey catalog (Bird et al. 2004). (b) BATSE Earth occultation catalog, Deep sample results (Harmon et al. 2004). (c) The first COMPTEL source catalogue (Schönfelder et al. 2000) (d) The third EGRET catalog of high-energy $\gamma$-ray sources (Hartman et al. 1999) (e) S: SIGMA instrument onboard GRANAT satellite; O: OSSE; T: TeV source 